\title{
Cyclic Variation in Heart Rate Score by Holter Electrocardiogram as Screening for Sleep-Disordered Breathing in Subjects With Heart Failure
}

\author{
Takeshi Shimizu MD, Akiomi Yoshihisa MD PhD, Shoji Iwaya MD, Satoshi Abe MD, \\ Takamasa Sato MD, Satoshi Suzuki MD PhD, Takayoshi Yamaki MD PhD, \\ Koichi Sugimoto MD PhD, Hiroyuki Kunii MD PhD, Kazuhiko Nakazato MD PhD, \\ Hitoshi Suzuki MD PhD, Shu-ichi Saitoh MD PhD, and Yasuchika Takeishi MD PhD
}

BACKGROUND: Sleep-disordered breathing (SDB) is critically associated with cardiovascular mortality and morbidity, especially in patients with heart failure (HF). However, the majority of SDB patients remain undiagnosed. In contrast, abnormality in heart rate variability has been reported in patients with SDB. To explore an efficient electrocardiogram (ECG)-based screening tool for SDB, we examined the usefulness of cyclic variation in heart rate score (CVHRS) by Holter ECG in subjects with HF. METHODS: In this study, 102 subjects with HF were enrolled. We simultaneously performed Holter ECG with overnight portable sleep monitoring, and we measured the respiratory disturbance index (RDI) and CVHRS. We determined the temporal position of the individual dips comprising the CVHRS using time-domain methods. CVHRS was measured as cyclic and autocorrelated dips in smoothed interbeat interval time series. RESULTS: There were 25 subjects with severe SDB (RDI $\geq 30$ events/h) and 77 subjects with none-to-moderate SDB $(0 \leq$ RDI $<30$ events/h). There was a significant positive correlation between CVHRS and RDI $(\mathrm{r}=0.60, P<.001)$. In receiver operating characteristic analysis, CVHRS (cutoff of 30 events/h) identified severe SDB with a sensitivity of $82 \%$, a specificity of $77 \%$, and an area under the curve of 0.83. CONCLUSIONS: CVHRS determined by Holter ECG is a useful screening index for severe SDB in subjects with HF. Key words: sleep-disordered breathing; heart failure; heart rate variability; ECG-based screening; apnea; hypoxia. [Respir Care 2015;60(1):72-80. (C) 2015 Daedalus Enterprises]

\section{Introduction}

Despite recent advancements in its medical management, heart failure (HF) still leads to high morbidity and mortality. The lifetime risk of developing $\mathrm{HF}$ is $20 \%$ at $\geq 40 \mathrm{y}$ of age, and the absolute mortality rate for $\mathrm{HF}$

Drs Shimizu, Yoshihisa, Iwaya, Abe, Sato, S Suzuki, Yamaki, Sugimoto, Kunii, Nakazato, H Suzuki, Saitoh, and Takeishi are affiliated with the Department of Cardiology and Hematology, and Drs Yoshihisa, S Suzuki, and Takeishi are affiliated with the Department of Advanced Cardiac Therapeutics, Fukushima Medical University, Fukushima, Japan.

Supplementary material related to this paper is available at http:// www.rcjournal.com.

This study was supported in part by Grant-in-Aid for Scientific Research 25461061 from the Japan Society for the Promotion of Science and remains $\sim 50 \%$ within 5 y of diagnosis. ${ }^{1}$ To improve survival, identification of factors that contribute to increased mortality is required. About $50 \%$ of patients with HF have sleep-disordered breathing (SDB), which consists of obstructive sleep apnea (OSA) and Cheyne-Stokes respiration with central sleep apnea. Some large-scale studies have demonstrated that SDB is associated with occurrence

\footnotetext{
grants-in-aid from the Japanese Ministry of Health, Labour, and Welfare (Tokyo, Japan). The Department of Advanced Cardiac Therapeutics is supported by Fukuda Denshi. The authors have disclosed no other conflicts of interest.

Correspondence: Akiomi Yoshihisa MD PhD, Department of Cardiology and Hematology, Fukushima Medical University, 1 Hikarigaoka, Fukushima 960-1295, Japan. E-mail: yoshihis@ fmu.ac.jp.
}

DOI: $10.4187 /$ respcare. 03341 
of ventricular arrhythmias ${ }^{2,3}$ and adverse prognosis in subjects with HF. ${ }^{4,5}$ Studies have demonstrated that treatment of SDB may improve prognosis in subjects with HF. ${ }^{6-9}$ However, because of the lack of patients' and physicians' awareness of SDB, especially in patients with HF, and limited access to portable sleep monitoring or overnight polysomnography, the majority of SDB patients remain undiagnosed. ${ }^{10}$ Javaheri et $\mathrm{al}^{10}$ reported that only $2 \%$ of subjects with HF received SDB testing. Thus, we are looking for alternative tools that are less labor-intensive and cheaper with better accessibility for patients with HF.

Abnormality in heart rate variability ${ }^{11}$ has been reported in subjects with $\mathrm{HF}$ and/or SDB. ${ }^{3,12,13} \mathrm{SDB}$ is associated with an altered sympathovagal balance determined by nocturnal cyclic alternating of apneas and hyperventilationbradycardia during apnea, followed by abrupt tachycardia. ${ }^{14}$ This phenomenon causes cyclic variation in heart rate (CVHR). ${ }^{14}$ Not only OSA but also Cheyne-Stokes respiration with central sleep apnea demonstrated heart rate oscillations. ${ }^{15}$ Both types of SDB present cyclic lengthening/shortening in R-R interval during apnea/post-apneic hyperventilation. ${ }^{16}$ Until now, this ordered variation in heart rate has been applied to the detection of SDB by only a few study groups for the general population who received polysomnography. ${ }^{17-20}$ Previous studies showed data for only OSA in the general population, excluding subjects with HF. Patients with HF show reduction in heart rate variability power, take $\beta$ blockers, and have complex SDB, including not only OSA but also Cheyne-Stokes respiration with central sleep apnea. Prevalence of Cheyne-Stokes respiration with central sleep apnea is increased with HF. ${ }^{21}$ Hence, techniques that could provide a simple screening tool for SDB are needed. The Holter electrocardiogram (ECG) is performed for many patients with HF. Screening for SDB by Holter ECG may be useful for many patients with HF without access to polysomnography. To explore an efficient Holter ECG-based screening tool for SDB in patients with $\mathrm{HF}$, we examined the usefulness of CVHR score (CVHRS) by Holter ECG.

\section{Methods}

\section{Study Subjects and Study Protocol}

In this study, we enrolled 110 consecutive subjects with HF who were referred for an overnight portable sleep monitor test with hospitalization regardless of SDB symptoms (such as daytime sleepiness determined by the Epworth sleepiness scale $^{22}$ ) at Fukushima Medical University. Inclusion criteria were: (1) presence of symptomatic HF, defined as New York Heart Association class $\geq \mathrm{II}^{1}$; (2) enforcement of standard pharmacotherapy (including angiotensin-converting enzyme inhibitors, angiotensin II receptor blockers, $\beta$ blockers, and diuretics); and (3) stable

\section{QUICK LOOK}

\section{Current knowledge}

Sleep apnea and sleep-disordered breathing (SDB) are associated with significant morbidity and mortality in patients with heart failure (HF). SDB in HF is also largely undiagnosed.

\section{What this paper contributes to our knowledge}

Cyclic variation in heart rate (CVHR) and respiratory disturbance index were significantly correlated in subjects with severe SDB. Receiver operating characteristic analysis demonstrated a high sensitivity and specificity for heart rate variation and SDB severity. Monitoring the CVHR score may be a useful screening index for SDB in HF.

clinical status, which was defined as receiving optimal medical therapy without worsening of HF for at least 2 months before study enrollment. Exclusion criteria were: (1) presence of atrial fibrillation or pacemaker implantation, (2) overnight recordings with $>10 \%$ noise or ectopic beats, (3) history of stroke with neurological deficit, (4) acute coronary syndrome and acute decompensated HF, and (5) recent SDB treatment. The final total was 102 subjects.

We performed simultaneous overnight portable sleep monitoring and Holter ECG monitoring. Standard Holter ECG recorders (LS-300, Fukuda Denshi, Tokyo, Japan) were used to acquire data. Polygraphic data and Holter ECG data were matched using polygraphic time synchronized with Holter ECG time before lights out. Two independent physicians, each one blinded to the results of the other, performed heart rate variability analysis and synchronized polygraphic scoring. Subjects were divided into 2 groups based on whether the respiratory disturbance index (RDI) was $>30$ events/h (severe SDB, $n=25$ ) or not (none-to-moderate $\mathrm{SDB}, n=77$ ) via portable sleep monitor. Written informed consent was obtained from all study subjects. The study protocol was approved by the ethics committee of Fukushima Medical University (number 1050).

\section{Portable Sleep Monitor}

All subjects underwent overnight polygraphy with standard techniques. ${ }^{23}$ An overnight sleep study was performed using the LS-300 cardiopulmonary monitoring system (type 3 polygraph). ECG, thoracoabdominal motion, nasal air flow via an air-flow pressure transducer, and $\mathrm{S}_{\mathrm{pO}_{2}}$ were monitored as reported previously. ${ }^{23}$ Apnea was defined as 
an absence of air flow for $>10 \mathrm{~s}$. Hypopnea was defined as a $>30 \%$ reduction in monitored air flow accompanied by a decrease in $\mathrm{S}_{\mathrm{pO}_{2}}$ of $>3 \% .{ }^{24}$ Standard definitions were used for OSA and central sleep apnea based on the presence or absence of rib cage and abdominal excursions with an absence of air flow. ${ }^{24}$ RDI was defined as the number of apneas and hypopneas/h during time spent in bed. ${ }^{24}$ The major polygraphic parameters investigated were RDI, central RDI, obstructive RDI, 3\% oxygen desaturation index (3\% ODI), lowest $\mathrm{S}_{\mathrm{pO}_{2}}$, and mean $\mathrm{S}_{\mathrm{pO}_{2}} \cdot{ }^{23}$ These data were visually inspected and scored by a single experienced laboratory technician who was blinded to the other results. Our preliminary data from another cohort $(n=16)$ showed that the RDI obtained with the LS-300 system closely correlated with the apnea-hypopnea index obtained by polysomnography $(\mathrm{r}=0.93, P<.01$; see the supplementary materials at http://www.rcjournal.com).

\section{ECG Analysis and CVHRS Measurement}

ECG signals were obtained via the Holter LS-300 system at a sampling frequency of $125 \mathrm{~Hz} .{ }^{25}$ A Holter automatic arrhythmia analysis system (SCM-8000, Fukuda Denshi) identified all R-wave positions and excluded abnormal beats, such as ventricular ectopic and supraventricular ectopic beats and artifacts. Moreover, the R-R interval of normal beats that exceeded $\pm 30 \%$ of the average $\mathrm{R}-\mathrm{R}$ interval was removed from the trend signal. Because the R-R interval time series data had irregular intervals after excluding all abnormal beats, a resampling signal process was performed at $2 \mathrm{~Hz}$ empirically. For automated detection of cyclic variation in heart rate, we developed a new algorithm. The methods used to create the parameters to detect dip candidates are shown in Figure 1. We created a differentiation waveform, as shown in Figure 1C, from R-R time series data $\mathrm{f}(\mathrm{i})$, which was obtained after the resampling signal process, in Figure 1B through a bandpass filter $(0.02-0.11 \mathrm{~Hz})$. Three points of intersection ( $\mathrm{x} 1$, $\mathrm{x} 2$, and $\mathrm{x} 3$ ) in Figure $1 \mathrm{C}$ and baselines were created as dip starting point $y 1$, lowest dip point $y 2$, and dip ending point $\mathrm{y} 3$. We defined $\mathrm{y} 1$ and $\mathrm{y} 3$ as the maximum points of $\mathrm{x} 1$ and $x 3$ in the vicinity of $3 \mathrm{~s}$ as $\mathrm{y} 2$ as the minimum point of $\mathrm{x} 2$ in the vicinity of $3 \mathrm{~s}$ (Fig. 1B).

Next, the height of dip h was calculated in each of the dip candidates as: $\mathrm{h}=0.8[(\mathrm{y} 1-\mathrm{y} 2)+(\mathrm{y} 3-\mathrm{y} 2)] / 2$ (Fig. 2B). In another cohort with no cardiovascular disease $(n=36)$, we postulated that dip $\mathrm{h}$ is calculated as: $\mathrm{h}=[(\mathrm{y} 1-\mathrm{y} 2)+(\mathrm{y} 3-\mathrm{y} 2)] / 2$. We then defined the width of dip $d$ as the interval points of $f(i)=h$, as shown in Figure 1B, whereas the true dip $d$ was defined as the inter-apnea interval (time during post-apneic hyperventilation). The coefficient was determined as 0.8 using the least-squares method in the other cohort. This value was adopted as the coefficient since the mean squared error
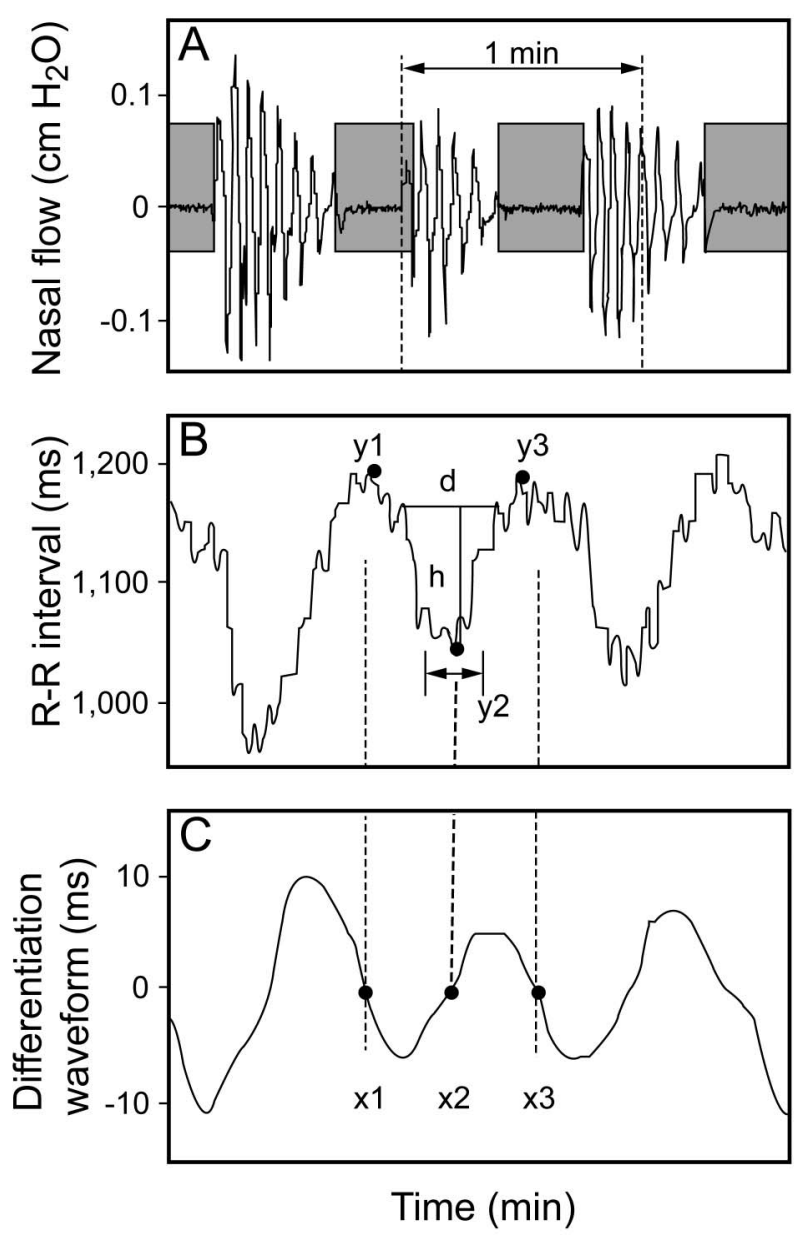

Fig. 1. A: Nasal flow. B: R-R interval. C: Differentiation waveform. A differentiation waveform was created from the $\mathrm{R}-\mathrm{R}$ time series data $f(i)(A)$ through a band-pass filter $(0.02-0.11 \mathrm{~Hz})$. The method used to detect dip candidate points is shown. $x 1, x 2$, and $x 3$ are zero cross points of the band-pass filter wave. $y 1, y 2$, and $y 3$ are the dip starting point, the lowest point on the dip, and the dip ending point, respectively.

was smallest when the parameter was 0.8 (see the supplementary materials at http://www.rcjournal.com). All h dips were measured from $\mathrm{f}(\mathrm{i})$. How significant dips were picked from dip candidates is shown in Figure 2. We determined the window width of each dip candidate as $6 \mathrm{~min}( \pm 3 \mathrm{~min}$ from the dip candidate) in which at least one cycle of long apnea duration could be included because the longest apnea duration seemed to be $3 \mathrm{~min}$ empirically. The mean $+1.5 \mathrm{SD}$ of dip $\mathrm{h}$ in a window of 6 min was defined as the threshold for the dips for excluding the dip candidates that might be respiratory changes (noises). To determine the SD coefficient as 1.5 , we calculated the CVHRS (events/h), changing the value of the SD coefficient from the database for developing this algorithm. We adopted 1.5 SD because the root mean square of sensitivity and specificity was highest with 1.5 as the SD coefficient using 

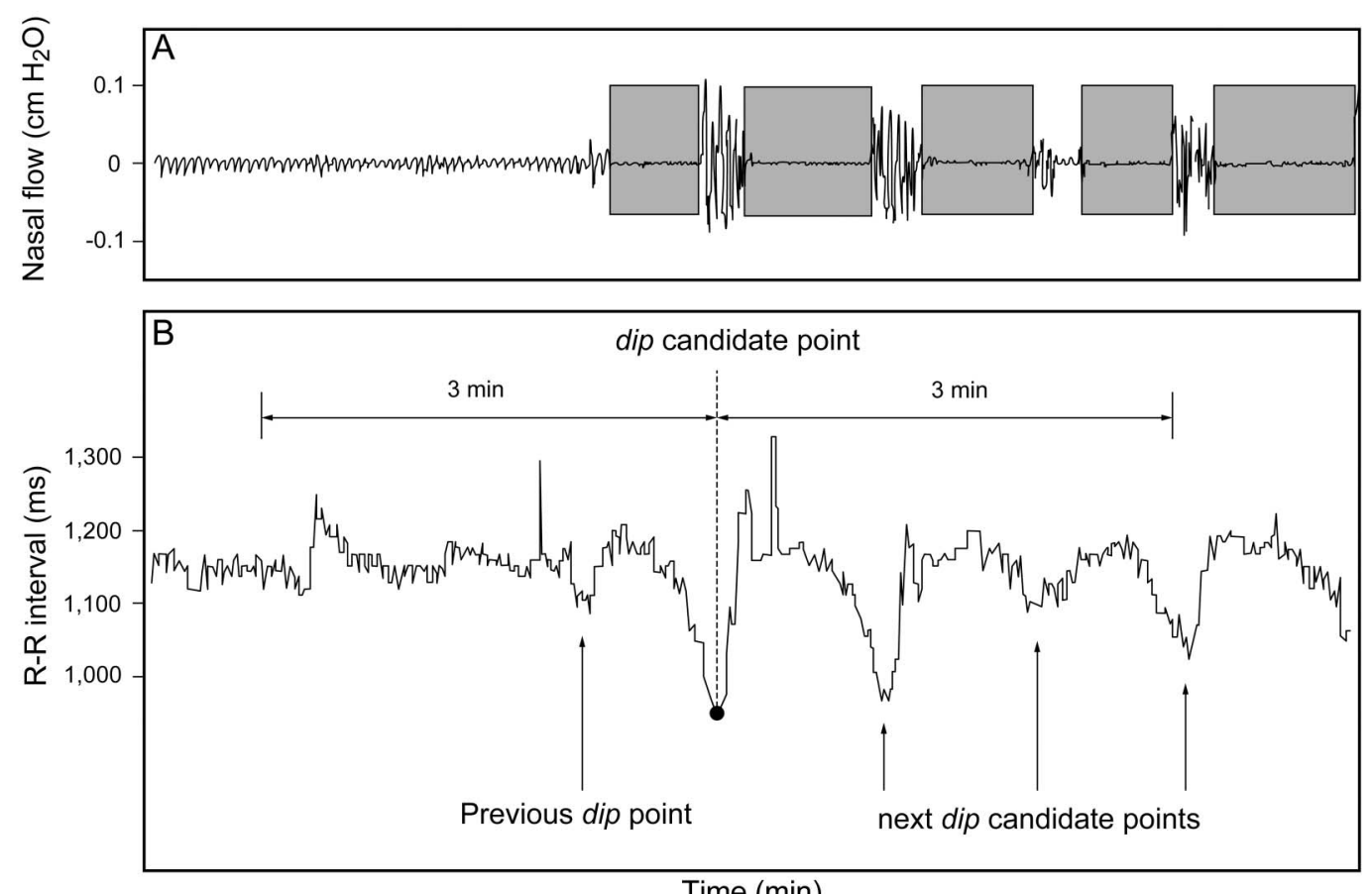

Time (min)

Fig. 2. A: Nasal flow. B: R-R interval. The calculation interval for determining a threshold value for the dip candidate point is shown.

the Youden index method in another cohort $(n=36$; see the supplementary materials at http://www.rcjournal.com). We calculated the mean $+1.5 \mathrm{SD}$ of 3 min before and after the vicinity of the dip candidate, and it served as the threshold for the dip candidates; when the height $\mathrm{h}$ in a candidate point exceeded the average of $+1.5 \mathrm{SD}$, this candidate point was recognized as the change from apnea to hyperventilation in the R-R time series data. Thus, we calculated the CVHRS as the mean number of CVHR/h of time in bed, which was defined by the behavior records of subjects. In the other cohort with no cardiovascular disease $(n=36)$, the CVHRS and RDI obtained with the LS-300 system closely correlated $(\mathrm{r}=0.86, P<.01$; see the supplementary materials at http://www.rcjournal.com).

\section{Measurement of Laboratory and Echocardiographic Data}

Blood samples were obtained the morning after polysomnography while subjects were in a supine position. Plasma brain natriuretic peptide levels were measured using a specific immunoradiometric assay (ShionoRIA BNP kit, Shionogi, Osaka, Japan). The estimated glomerular filtration rate was measured using the Modification of Diet in Renal Disease formula. ${ }^{26}$ Echocardiography was performed using standard techniques by an experienced echocardiographer at the echo laboratory in our hospital during the day. Echocardiographic parameters investigated included left ventricular ejection fraction and right ventric- ular fractional area change. ${ }^{27}$ The left ventricular ejection fraction was calculated using a modification of Simpson's method. The right ventricular fractional area change, defined as (end diastolic area - end systolic area)/end diastolic area $\times 100$, is a measure of right ventricular systolic function. ${ }^{27}$ All recordings were performed using ultrasound systems (Acuson Sequoia, Siemens, Mountain View, California).

\section{Statistical Analysis}

The Kolmogorov-Smirnov test was used for assessment of normal distribution. Normally distributed data are presented as mean $\pm \mathrm{SD}$, and non-normally distributed data are presented as the median (interquartile range). Categorical variables are expressed as frequencies and relative frequencies. Characteristics between the 2 groups were compared using the independent Student $t$ test for normally distributed data and the Mann-Whitney $U$ test for non-normally distributed data for continuous variables, whereas the chi-square test was used for categorical variables. Correlations between the RDI and CVHRS were assessed using Pearson correlation analysis. $P<.05$ was considered significant. To assess the ability of the CVHRS to correctly rank order subjects according to detected severe SDB, we measured the area under the receiver operating characteristic curve. The outcome we detected was severe SDB. These analyses were performed using SPSS 21.0 (IBM, Armonk, New York). 

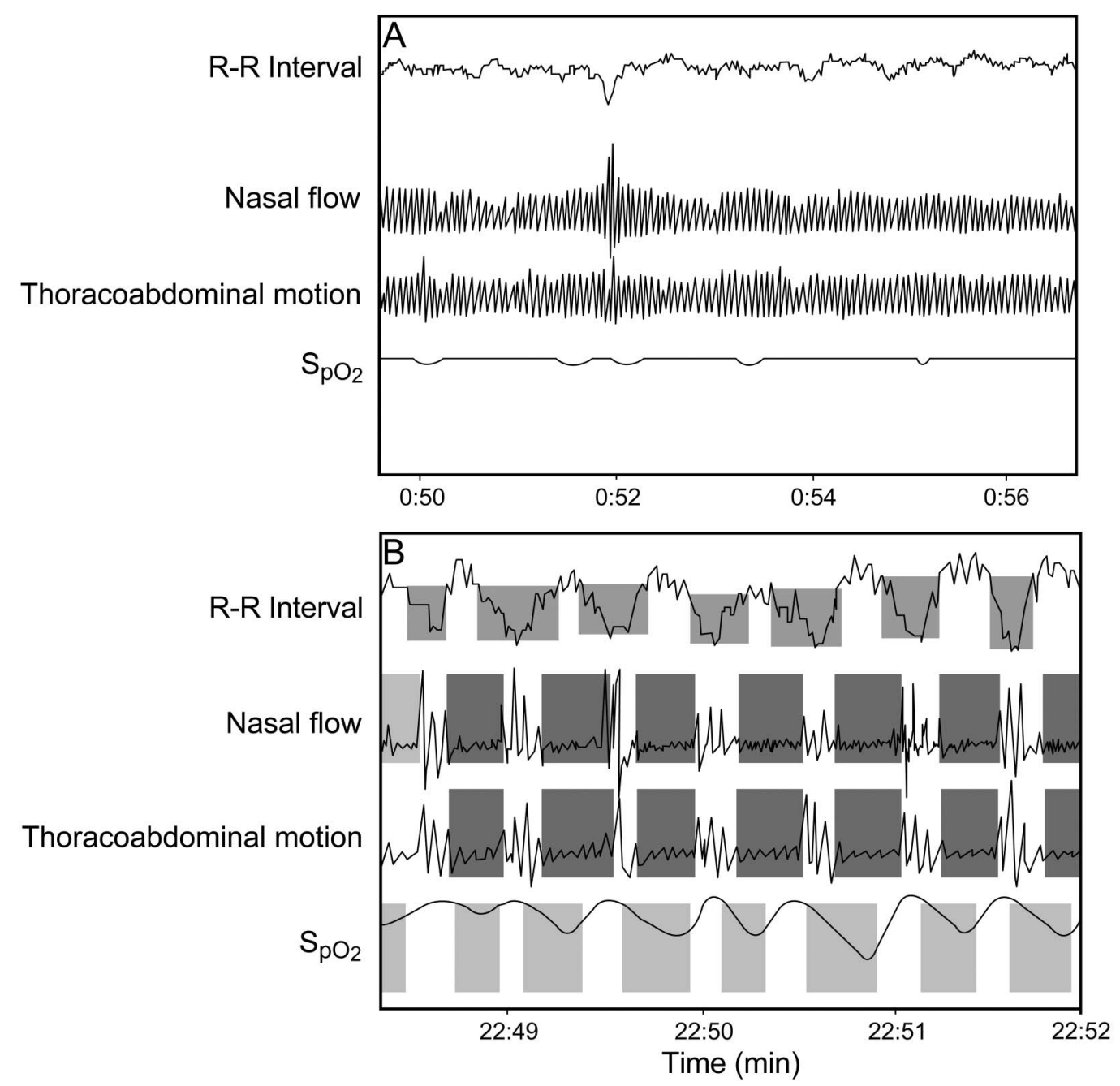

Fig. 3. Recordings of R-R interval, nasal flow, thoracoabdominal motion, and $\mathrm{S}_{\mathrm{pO}_{2}}$ obtained by polysomnography and Holter electrocardiography. A: subject with heart failure (HF) and mild sleep-disordered breathing (SDB): respiratory disturbance index (RDI) of 7.2 events/h and cyclic variation in heart rate score (CVHRS) of 6.3 events/h. B: subject with HF and severe SDB: RDI of 86.9 events/h and CVHRS of 70.9 events/h.

\section{Results}

\section{Case Presentation}

Figure 3 shows a subject's polygraph and Holter ECG record, including R-R interval, nasal air flow, thoracoabdominal motion, and $\mathrm{S}_{\mathrm{pO}_{2}}$. SDB subjects showed cyclic fluctuations in heart rate, consisting of bradycardia during apnea followed by tachycardia upon its cessation, which is called CVHR. CVHR makes dips in R-R interval series. Figure $3 \mathrm{~A}$ shows recordings from a subject with HF and mild SDB (RDI of 7.2 events/h and CVHRS of 6.3 events/h). Figure 3B shows those from a subject with HF and severe SDB (RDI of 86.9 events/h and CVHRS of 70.9 events/h).

\section{Clinical Characteristics of Study Subjects}

Twenty-five subjects had severe SDB (RDI $\geq 30$ events/h), and 77 subjects had none-to-moderate $\mathrm{SDB}(0 \leq \mathrm{RDI}<30$ events/h). Clinical characteristics of subjects with severe SDB and none-to-moderate SDB are compared in Table 1. Standard pharmacotherapy, including angiotensinconverting enzyme inhibitors, angiotensin II receptor blockers, and $\beta$ blockers, were given to the majority of subjects. There were no significant differences in age, gender, medications, laboratory data, and echocardiographic data between the 2 groups. The RDI, central RDI, and obstructive RDI were significantly higher, whereas the lowest $\mathrm{S}_{\mathrm{pO}_{2}}$ and mean $\mathrm{S}_{\mathrm{pO}_{2}}$ were significantly lower in severe $\mathrm{SDB}$ group compared with the none-to-moderate SDB group. 
Table 1. Clinical Characteristics

\begin{tabular}{|c|c|c|c|}
\hline & $\begin{array}{l}\text { Severe SDB } \\
\quad(n=25)\end{array}$ & $\begin{array}{l}\text { None-to-moderate SDB } \\
\quad(n=77)\end{array}$ & $P$ \\
\hline Age (mean $\pm S D), y$ & $62.6 \pm 12.3$ & $56.8 \pm 14.1$ & .07 \\
\hline Male, $n(\%)$ & $19(76.0)$ & $60(77.9)$ & .84 \\
\hline NYHA functional class (I/II/III/IV) & $0 / 12 / 12 / 1$ & $0 / 44 / 30 / 3$ & .72 \\
\hline Body mass index (mean $\pm \mathrm{SD}), \mathrm{kg} / \mathrm{m}^{2}$ & $25.4 \pm 5.4$ & $24.4 \pm 4.2$ & .31 \\
\hline Systolic blood pressure (mean $\pm \mathrm{SD}$ ), $\mathrm{mm} \mathrm{Hg}$ & $119.1 \pm 15.6$ & $120.4 \pm 16.8$ & .78 \\
\hline Diastolic blood pressure (mean $\pm \mathrm{SD}$ ), $\mathrm{mm} \mathrm{Hg}$ & $73.4 \pm 10.5$ & $73.3 \pm 11.9$ & .96 \\
\hline Heart rate (mean $\pm \mathrm{SD})$, beats $/ \mathrm{min}$ & $69.8 \pm 17.5$ & $65.7 \pm 12.4$ & .33 \\
\hline Ischemic etiology, $n(\%)$ & $9(36.0)$ & $28(36.4)$ & .97 \\
\hline \multicolumn{4}{|l|}{ Medication, $n(\%)$} \\
\hline ACE inhibitors/ARB & $18(72.0)$ & $56(72.7)$ & .94 \\
\hline$\beta$ blockers & $20(80.0)$ & $63(81.8)$ & .84 \\
\hline Diuretics & $18(72.0)$ & $39(50.6)$ & .07 \\
\hline \multicolumn{4}{|l|}{ Data } \\
\hline Hemoglobin $($ mean $\pm \mathrm{SD}), \mathrm{g} / \mathrm{L}$ & $129 \pm 17$ & $136 \pm 20$ & .09 \\
\hline $\mathrm{P}_{\mathrm{aO}_{2}}($ mean $\pm \mathrm{SD}), \mathrm{mm} \mathrm{Hg}$ & $87.2 \pm 30.1$ & $95.7 \pm 27.9$ & .28 \\
\hline $\mathrm{P}_{\mathrm{aCO}_{2}}($ mean $\pm \mathrm{SD}), \mathrm{mm} \mathrm{Hg}$ & $38.0 \pm 7.3$ & $40.7 \pm 10.0$ & .29 \\
\hline $\mathrm{BNP}$ (median [IQR]), pg/mL & $196.4(32.1-398.8)$ & $87.8(17.0-346.8)$ & .25 \\
\hline Estimated glomerular filtration (mean $\pm \mathrm{SD}$ ), $\mathrm{mL} / \mathrm{min} / 1.73 \mathrm{~cm}^{2}$ & $65.0 \pm 29.4$ & $69.2 \pm 28.3$ & .58 \\
\hline C-reactive protein (median $[\mathrm{IQR}]$ ), $\mathrm{mg} / \mathrm{dL}$ & $0.25(0.06-0.98)$ & $0.11(0.04-0.64)$ & .22 \\
\hline Triglyceride (mean $\pm \mathrm{SD}), \mathrm{mg} / \mathrm{dL}$ & $109.4 \pm 44.8$ & $140.2 \pm 97.6$ & .24 \\
\hline Total cholesterol (mean $\pm \mathrm{SD}), \mathrm{mg} / \mathrm{dL}$ & $166.5 \pm 38.9$ & $185.6 \pm 47.6$ & .22 \\
\hline Low-density cholesterol (mean $\pm \mathrm{SD}$ ), $\mathrm{mg} / \mathrm{dL}$ & $99.7 \pm 27.5$ & $112.2 \pm 39.0$ & .25 \\
\hline High-density cholesterol (mean $\pm \mathrm{SD}$ ), $\mathrm{mg} / \mathrm{dL}$ & $47.9 \pm 15.0$ & $46.5 \pm 13.2$ & .72 \\
\hline Fasting blood glucose (mean $\pm \mathrm{SD}), \mathrm{mg} / \mathrm{dL}$ & $151.9 \pm 89.5$ & $127.9 \pm 59.3$ & .17 \\
\hline Hemoglobin A1c (mean $\pm \mathrm{SD}), \%$ & $6.4 \pm 1.9$ & $5.6 \pm 1.3$ & .11 \\
\hline $\mathrm{LVEF}($ mean $\pm \mathrm{SD}), \%$ & $50.1 \pm 15.1$ & $52.8 \pm 13.2$ & .46 \\
\hline RV-FAC (mean $\pm \mathrm{SD}$ ), $\%$ & $39.9 \pm 14.6$ & $41.8 \pm 11.5$ & .74 \\
\hline Epworth Sleepiness Scale (mean \pm SD) & $6.5 \pm 4.3$ & $5.6 \pm 3.8$ & .36 \\
\hline \multicolumn{4}{|l|}{ Polysomnography } \\
\hline RDI (mean $\pm \mathrm{SD})$, events $/ \mathrm{h}$ & $46.1 \pm 12.9$ & $15.7 \pm 7.3$ & $<.01$ \\
\hline Central RDI (median [IQR]), events/h & $17.6(10.4-28.6)$ & $2.1(0.9-4.3)$ & $<.01$ \\
\hline Obstructive RDI (median [IQR]), events/h & $14.0(3.3-26.5)$ & $3.7(0.5-9.3)$ & $<.01$ \\
\hline$>3 \%$ oxygen desaturation index (mean $\pm \mathrm{SD}$ ), events $/ \mathrm{h}$ & $41.6 \pm 14.2$ & $13.4 \pm 7.1$ & $<.01$ \\
\hline Lowest $\mathrm{S}_{\mathrm{pO}_{2}}($ mean $\pm \mathrm{SD}), \%$ & $75.2 \pm 10.3$ & $84.5 \pm 5.9$ & $<.01$ \\
\hline Mean $\mathrm{S}_{\mathrm{pO}_{2}}($ mean $\pm \mathrm{SD}), \%$ & $94.2 \pm 3.0$ & $96.6 \pm 2.5$ & $<.01$ \\
\hline SDB severity (none/mild/moderate/severe) & $0 / 0 / 0 / 25$ & $6 / 27 / 44 / 0$ & \\
\hline $\begin{array}{l}n=102 . \\
\text { SDB }=\text { sleep-disordered breathing } \\
\text { IQR = interquartile range } \\
\text { NYHA = New York Heart Association } \\
\text { ACE = angiotensin-converting enzyme } \\
\text { ARB = angiotensin II receptor blocker } \\
\text { BNP = brain natriuretic peptide } \\
\text { LVEF = left ventricular ejection fraction } \\
\text { RV-FAC = right ventricular fractional area change } \\
\text { RDI = respiratory disturbance index }\end{array}$ & & & \\
\hline
\end{tabular}

\section{Correlation Between Polygraphic Data and CVHRS}

As shown in Figure 4, there was a significant positive correlation between CVHRS and RDI $(\mathrm{r}=0.60, P<.001)$. As shown in Figure 5, receiver operating characteristic analysis revealed that the CVHRS (cutoff of 30 events/h) identified severe SDB with a sensitivity of $82 \%$, a speci- ficity of $77 \%$, and an area under the curve of 0.83 (95\% CI 0.72-0.93).

\section{Discussion}

In this study, CVHRS obtained with our automated CVHR detection algorithm showed good performance in 


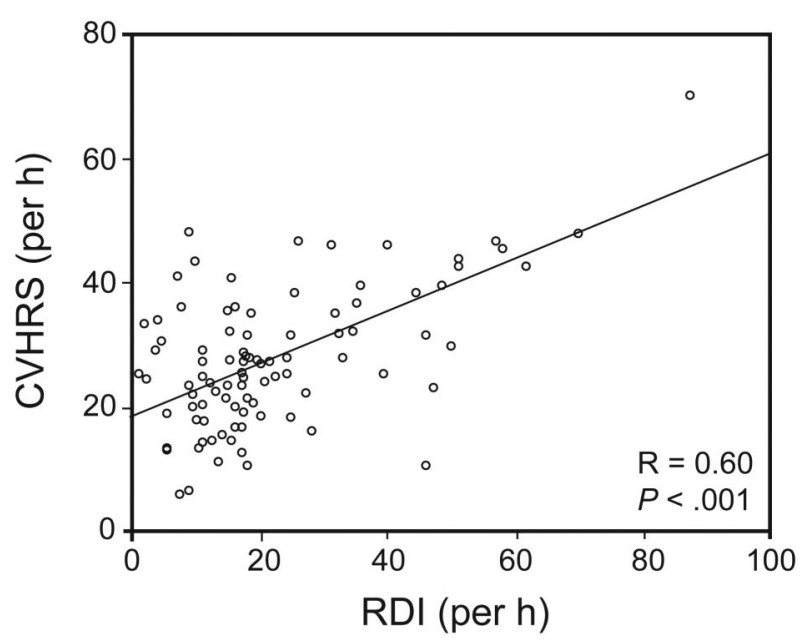

Fig. 4. Correlation between cyclic variation in heart rate score (CVHRS) and respiratory disturbance index (RDI) in subjects with heart failure.

identifying severe SDB in subjects with HF. To our knowledge, this is the first study to show that CVHRS is an excellent screening tool to detect severe SDB even in subjects with HF who have a reduction in power of heart rate variability, have taken $\beta$ blockers, and have complex SDB, including OSA and Cheyne-Stokes respiration with central sleep apnea.

\section{Impact of SDB on Hemodynamic Parameters and Heart Rate Variability in HF}

SDB (especially OSA) is characterized by recurrent hypoxia, arousal, and generation of exaggerated negative intrathoracic pressure, which increases sympathetic nervous activity, reduces cardiac parasympathetic activity, and causes repetitive surges in heart rate, blood pressure, and left ventricular preload and afterload, resulting in decreasing stroke volume. ${ }^{28}$ Yumino et $\mathrm{al}^{28}$ reported the different hemodynamic impact of OSA and Cheyne-Stokes respiration with central sleep apnea on subjects with HF: whereas OSA appears to have a decreasing stroke volume, CheyneStokes respiration with central sleep apnea seems to have little or slightly increased stroke volume. With respect to heart rate, not only OSA but also Cheyne-Stokes respiration with central sleep apnea demonstrated heart rate oscillations. ${ }^{15}$ Both types of SDB presented cyclic lengthening/shortening in the R-R interval during apnea/post-apneic hyperventilation. ${ }^{16}$ OSA and Cheyne-Stokes respiration with central sleep apnea are associated with a different autonomic control of heart rate. The respiratory component of the R-R interval variability (respiratory sinus arrhythmia) is reportedly highest during the apneic phase of OSA and is blunted during hyperventilation. Conversely, in central sleep apnea, the respiratory component of R-R

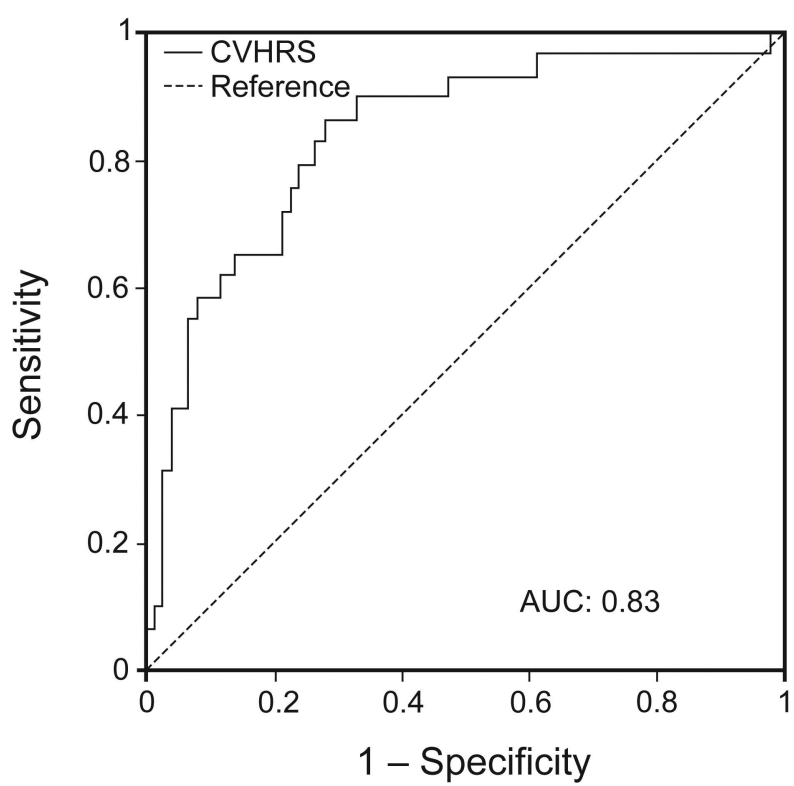

Fig. 5. Receiver operating characteristic curve to identify severe sleep-disordered breathing in subjects with heart failure. The area under the receiver operating characteristic curve (AUC) was 0.83 (95\% Cl 0.72-0.93). CVHRS = cyclic variation in heart rate score.

interval variability is nearly absent during the apneic phase and recovers with resumption of ventilation. ${ }^{16}$ Because of these features, episodes of Cheyne-Stokes respiration with central sleep apnea may be detected more consistently with the CVHRS compared with episodes of OSA; this may have led to the tendency for overestimation in subjects with Cheyne-Stokes respiration with central sleep apnea. ${ }^{15,29-31}$

\section{ECG-Based SDB Screening in Subjects With HF}

Cyclic variations in heart rate index accompanying SDB driven by respiratory control dysfunction are known to show more variable and irregular patterns of oscillations. ${ }^{32}$ Recently, several authors have developed algorithms for automated ECG detection of OSA in the general population using heart rate variability, including time and frequency domain analysis of R-R intervals, and some studies have reported that the combination of parameters of heart rate variability and ECG-derived respiration signals gave the best classification result. ${ }^{17-20}$ Roche et al ${ }^{33}$ proposed an increase in the relative power of the very-low-frequency component $(0.01-0.05 \mathrm{~Hz})$ of interbeat interval increment as a marker for OSA. They reported an area under the curve of 0.70 for identifying subjects with moderate SDB with $64 \%$ sensitivity and $69 \%$ specificity. Hayano et al ${ }^{34}$ used a time-domain and dip-detection method and algorithm (which they called ACAT algorithms) in the general population. The authors reported an area under the curve 


\section{Sleep-Disordered Breathing in Subjects With Heart Failure}

of 0.91 for identifying subjects with moderate SDB with $83 \%$ sensitivity and $88 \%$ specificity.

In patients with $\mathrm{HF}$, the power of heart rate variability decreases and is associated with adverse prognosis. ${ }^{35,36}$ There is convincing evidence that autonomic nervous imbalance caused by SDB contributes to increased mortality in patients with HF. ${ }^{37}$ Heart rate variability powers in subjects with HF and SDB were reportedly decreased during a 24-h period by increased sympathetic activity and damped circadian oscillation. ${ }^{3,38}$ Furthermore, patients with HF take $\beta$ blockers, which affect heart rate variability. Our study differs from previous studies in several ways. (1) We presented a new algorithm named CVHRS, and (2) our study population consisted of subjects with HF, who have a reduction in heart rate variability power, take $\beta$ blockers, and have complex SDB, including OSA and Cheyne-Stokes respiration with central sleep apnea.

\section{Limitations}

Our study has some limitations. First, subjects with atrial fibrillation were excluded because we could not determine the CVHRS in the presence of atrial fibrillation. Second, the influence of medical treatment on these study findings remains uncertain. Moreover, a previous study has shown that $\beta$ blockers change autonomic nervous balance in subjects with HF. ${ }^{39}$ Third, the accepted standard for diagnostic testing of SDB is full-channel polysomnography, which provides detailed information on complete differentiation of the types of apnea. Our differentiation of SDB using a portable sleep monitor might be less reliable than that by full-channel polysomnography. Thus, further investigation by full-channel polysomnography may lead to complete clarification of CVHRS in subjects with HF and SDB. Fourth, we cannot exclude the effect of periodic leg movements, which may affect heart rate variability. ${ }^{34,40}$ Periodic leg movements are characterized by recurrent episodes of repetitive stereotyped limb movements that occur predominantly in the legs. ${ }^{40}$ Finally, the sample size was relatively small. Large-scale clinical trials may be required to confirm the CVHRS algorithm to determine SDB in subjects with HF.

\section{Conclusions}

SDB screening by Holter ECG may be useful for many patients with HF who do not have access to polysomnography. CVHRS determined by Holter ECG is a useful screening index for severe SDB in patients with HF. We do not consider CVHRS to be a final diagnostic tool, but rather a screening tool for SDB. We hope that patients with HF and suspected SDB screened by Holter ECG will then be able to undergo the more precise polysomnography.

\section{ACKNOWLEDGMENTS}

We thank Ms Yuko Niimura for outstanding technical assistance.

\section{REFERENCES}

1. Writing Committee Members, Yancy CW, Jessup M, Bozkurt B, Butler J, Casey DE Jr, et al. 2013 ACCF/AHA guideline for the management of heart failure: a report of the American College of Cardiology Foundation/American Heart Association Task Force on practice guidelines. Circulation 2013;128(16):e240-e327.

2. Bitter T, Westerheide N, Prinz C, Hossain MS, Vogt J, Langer C, et al. Cheyne-Stokes respiration and obstructive sleep apnoea are independent risk factors for malignant ventricular arrhythmias requiring appropriate cardioverter-defibrillator therapies in patients with congestive heart failure. Eur Heart J 2011;32(1):61-74.

3. Yamada S, Suzuki H, Kamioka M, Suzuki S, Kamiyama Y, Yoshihisa A, et al. Sleep-disordered breathing increases risk for fatal ventricular arrhythmias in patients with chronic heart failure. Circ J 2013;77(6): 1466-1473.

4. Javaheri S, Shukla R, Zeigler H, Wexler L. Central sleep apnea, right ventricular dysfunction, and low diastolic blood pressure are predictors of mortality in systolic heart failure. J Am Coll Cardiol 2007; 49(20):2028-2034.

5. Wang H, Parker JD, Newton GE, Floras JS, Mak S, Chiu KL, et al. Influence of obstructive sleep apnea on mortality in patients with heart failure. J Am Coll Cardiol 2007;49(15):1625-1631.

6. Koyama T, Watanabe H, Igarashi G, Terada S, Makabe S, Ito H. Short-term prognosis of adaptive servo-ventilation therapy in patients with heart failure. Circ J 2011;75(3):710-712.

7. Miyata M, Yoshihisa A, Suzuki S, Yamada S, Kamioka M, Kamiyama Y, et al. Adaptive servo ventilation improves Cheyne-Stokes respiration, cardiac function, and prognosis in chronic heart failure patients with cardiac resynchronization therapy. J Cardiol 2012;60(3): 222-227.

8. Owada T, Yoshihisa A, Yamauchi H, Iwaya S, Suzuki S, Yamaki T, et al. Adaptive servoventilation improves cardiorenal function and prognosis in heart failure patients with chronic kidney disease and sleep-disordered breathing. J Card Fail 2013;19(4):225-232.

9. Yoshihisa A, Suzuki S, Yamaki T, Sugimoto K, Kunii H, Nakazato $\mathrm{K}$, et al. Impact of adaptive servo-ventilation on cardiovascular function and prognosis in heart failure patients with preserved left ventricular ejection fraction and sleep-disordered breathing. Eur J Heart Fail 2013;15(5):543-550.

10. Javaheri S, Caref EB, Chen E, Tong KB, Abraham WT. Sleep apnea testing and outcomes in a large cohort of Medicare beneficiaries with newly diagnosed heart failure. Am J Respir Crit Care Med 2011; 183(4):539-546.

11. Heart rate variability. Standards of measurement, physiological interpretation, and clinical use. Task Force of the European Society of Cardiology and the North American Society of Pacing and Electrophysiology. Eur Heart J 1996;17(3):354-381.

12. Szollosi I, Krum H, Kaye D, Naughton MT. Sleep apnea in heart failure increases heart rate variability and sympathetic dominance. Sleep 2007;30(11):1509-1514.

13. Abdullah H, Maddage NC, Cosic I, Cvetkovic D. Cross-correlation of EEG frequency bands and heart rate variability for sleep apnoea classification. Med Biol Eng Comput 2010;48(12):1261-1269.

14. Guilleminault C, Connolly S, Winkle R, Melvin K, Tilkian A. Cyclical variation of the heart rate in sleep apnoea syndrome. Mechanisms, and usefulness of $24 \mathrm{~h}$ electrocardiography as a screening technique. Lancet 1984;1(8369):126-131.

15. Leung RS, Floras JS, Lorenzi-Filho G, Rankin F, Picton P, Bradley TD. Influence of Cheyne-Stokes respiration on cardiovascular oscil- 


\section{Sleep-Disordered Breathing in Subjects With Heart Failure}

lations in heart failure. Am J Respir Crit Care Med 2003;167(11): 1534-1539.

16. Spicuzza L, Bernardi L, Calciati A, Di Maria GU. Autonomic modulation of heart rate during obstructive versus central apneas in patients with sleep-disordered breathing. Am J Respir Crit Care Med 2003;167(6):902-910.

17. Penzel T, McNames J, Murray A, de Chazal P, Moody G, Raymond B. Systematic comparison of different algorithms for apnoea detection based on electrocardiogram recordings. Med Biol Eng Comput 2002;40(4):402-407.

18. Khandoker AH, Palaniswami M, Karmakar CK. Support vector machines for automated recognition of obstructive sleep apnea syndrome from ECG recordings. IEEE Trans Inf Technol Biomed 2009; 13(1):37-48

19. Mendez MO, Corthout J, Van Huffel S, Matteucci M, Penzel T, Cerutti S, Bianchi AM. Automatic screening of obstructive sleep apnea from the ECG based on empirical mode decomposition and wavelet analysis. Physiol Meas 2010;31(3):273-289.

20. Babaeizadeh S, White DP, Pittman SD, Zhou SH. Automatic detection and quantification of sleep apnea using heart rate variability. $\mathrm{J}$ Electrocardiol 2010;43(6):535-541.

21. Somers VK, White DP, Amin R, Abraham WT, Costa F, Culebras A, et al. Sleep apnea and cardiovascular disease. An American Heart Association/American College of Cardiology Foundation Scientific Statement from the American Heart Association Council for High Blood Pressure Research Professional Education Committee, Council on Clinical Cardiology, Stroke Council, and Council on Cardiovascular Nursing in collaboration with the National Heart, Lung, and Blood Institute National Center on Sleep Disorders Research (National Institutes of Health). Circulation 2008;118(10):1080-1111.

22. Miletin MS, Hanly PJ. Measurement properties of the Epworth sleepiness scale. Sleep Med 2003;4(3):195-199.

23. Silber MH, Ancoli-Israel S, Bonnet MH, Chokroverty S, GriggDamberger MM, Hirshkowitz M, et al. The visual scoring of sleep in adults. J Clin Sleep Med 2007;3(2):121-131.

24. Berry RB, Budhiraja R, Gottlieb DJ, Gozal D, Iber C, Kapur VK, et al. Rules for scoring respiratory events in sleep: update of the 2007 AASM Manual for the Scoring of Sleep and Associated Events. Deliberations of the Sleep Apnea Definitions Task Force of the American Academy of Sleep Medicine. J Clin Sleep Med 2012;8(5): 597-619.

25. Bianchi AM, Mainardi L, Petrucci E, Signorini MG, Mainardi M, Cerutti S. Time-variant power spectrum analysis for the detection of transient episodes in HRV signal. IEEE Trans Biomed Eng 1993; 40(2):136-144.

26. Levey AS, Coresh J, Greene T, Stevens LA, Zhang YL, Hendriksen $\mathrm{S}$, et al. Using standardized serum creatinine values in the modification of diet in renal disease study equation for estimating glomerular filtration rate. Ann Intern Med 2006;145(4):247-254.
27. Rudski LG, Lai WW, Afilalo J, Hua L, Handschumacher MD, Chandrasekaran K, et al. Guidelines for the echocardiographic assessment of the right heart in adults: a report from the American Society of Echocardiography endorsed by the European Association of Echocardiography, a registered branch of the European Society of Cardiology, and the Canadian Society of Echocardiography. J Am Soc Echocardiogr 2010;23(7):685-713.

28. Yumino D, Kasai T, Kimmerly D, Amirthalingam V, Floras JS, Bradley TD. Differing effects of obstructive and central sleep apneas on stroke volume in patients with heart failure. Am J Respir Crit Care Med 2013;187(4):433-438.

29. Franklin KA, Sandström E, Johansson G, Bâlfors EM. Hemodynamics, cerebral circulation, and oxygen saturation in Cheyne-Stokes respiration. J Appl Physiol 1997;83(4):1184-1191.

30. Thomas RJ, Mietus JE, Peng CK, Gilmartin G, Daly RW, Goldberger AL, Gottlieb DJ. Differentiating obstructive from central and complex sleep apnea using an automated electrocardiogram-based method. Sleep 2007;30(12):1756-1769.

31. Yagishita-Tagawa Y, Yumino D, Takagi A, Serizawa N, Hagiwara $\mathrm{N}$. Association between sleep apnea and overnight hemodynamic changes in hospitalized heart failure patients with and without paroxysmal nocturnal dyspnea. J Cardiol 2013;61(5):348-353.

32. Badr MS. Central sleep apnea. Prim Care 2005;32(2):361-374.

33. Roche F, Celle S, Pichot V, Barthélémy JC, Sforza E. Analysis of the interbeat interval increment to detect obstructive sleep apnoea/hypopnoea. Eur Respir J 2007;29(6):1206-1211.

34. Hayano J, Watanabe E, Saito Y, Sasaki F, Fujimoto K, Nomiyama T, et al. Screening for obstructive sleep apnea by cyclic variation of heart rate. Circ Arrhythm Electrophysiol 2011;4(1):64-72.

35. Fauchier L, Babuty D, Cosnay P, Autret ML, Fauchier JP. Heart rate variability in idiopathic dilated cardiomyopathy: characteristics and prognostic value. J Am Coll Cardiol 1997;30(4):1009-1014.

36. van de Borne P, Montano N, Pagani M, Oren R, Somers VK. Absence of low-frequency variability of sympathetic nerve activity in severe heart failure. Circulation 1997;95(6):1449-1454.

37. Hirsch AT, Dzau VJ, Creager MA. Baroreceptor function in congestive heart failure: effect on neurohumoral activation and regional vascular resistance. Circulation 1987;75(5 Pt 2):IV36-IV48.

38. Ueno LM, Drager LF, Rodrigues AC, Rondon MU, Mathias W Jr, Krieger EM, et al. Day-night pattern of autonomic nervous system modulation in patients with heart failure with and without sleep apnea. Int J Cardiol 2011;148(1):53-58.

39. Tamura A, Kawano Y, Naono S, Kotoku M, Kadota J. Relationship between beta-blocker treatment and the severity of central sleep apnea in chronic heart failure. Chest 2007;131(1):130-135.

40. Sasai T, Matsuura M, Inoue Y. Change in heart rate variability precedes the occurrence of periodic leg movements during sleep: an observational study. BMC Neurol 2013;13(1):139. 